\title{
Dynamics of Satellites Around Asteroids in Presence of Solar Radiation Pressure
}

\author{
Xiaosheng Xin ${ }^{1}$, Daniel J. Scheeres ${ }^{2}$, Xiyun $\mathrm{Hou}^{3}$ and Lin Liu ${ }^{4}$ \\ ${ }^{1}$ School of Astronomy and Space Science, Nanjing University, \\ 163 Xianlin Avenue, Nanjing, Jiangsu 210023, China \\ email: xiaoshengxin@outlook.com \\ ${ }^{2}$ Department of Aerospace Engineering Sciences, University of Colorado at Boulder, \\ 429 UCB, Boulder, CO 80309, USA \\ email: scheeres@colorado.edu \\ ${ }^{3}$ School of Astronomy and Space Science, Nanjing University, \\ 163 Xianlin Avenue, Nanjing, Jiangsu 210023, China \\ email: silence@nju.edu.cn \\ ${ }^{4}$ School of Astronomy and Space Science, Nanjing University, \\ 163 Xianlin Avenue, Nanjing, Jiangsu 210023, China \\ email: lliu@nju.edu.cn
}

\begin{abstract}
Due to the close distance to the Sun, solar radiation pressure (SRP) plays an important role in the dynamics of satellites around near-Earth asteroids (NEAs). In this paper, we focus on the equilibrium points of a satellite orbiting around an asteroid in presence of SRP in the asteroid rotating frame. The asteroid is modelled as a uniformly rotating triaxial ellipsoid. When SRP comes into play, the equilibrium points transformed into periodic orbits termed as "dynamical substitutes". We obtain the analytical approximate solutions of the dynamical substitutes from the linearised equations of motion. The analytical solutions are then used as initial guesses and are numerically corrected to compute the accurate orbits of the dynamical substitutes. The stability of the dynamical substitutes is analysed and the stability maps are obtained by varying parameters of the ellipsoid model as well as the magnitude of SRP.
\end{abstract}

Keywords. Minor planets, asteroids; Methods: numerical

\section{Introduction}

Asteroids generally have small masses and irregular shapes compared to planetary objects. As a good approximation, the asteroid can be modelled as a uniformly rotating triaxial ellipsoid. When analysing satellite motion around an asteroid in the asteroid rotating frame within this model, Scheeres (1994) shows that there are two types of equilibrium points in the rotation plane, i.e., saddle points, which lie on the long axis of the ellipsoid and are invariably unstable, and center points, which lie on the short axis of the ellipsoid and their stability are closely related to the shape of the ellipsoid and its rotation period.

For the near-Earth asteroid (NEA), solar radiation pressure (SRP) also plays an important role and has strong influence on the dynamics of an orbiting satellite. Morrow et al. (2001) analyse the hovering points around the asteroid utilising SRP and Scheeres (2012) presents two types of frozen orbits around the asteroid considering SRP. Under the perturbation of SRP, the dynamical system becomes time-dependent and the equilibrium points discussed by Scheeres (1994) no longer exist. They turn into dynamical substitutes which form closed periodic orbits around the equilibrium points. In this paper, we analyse those dynamical substitutes and their stability. 


\section{Dynamical Model}

The dynamical model is constructed in the asteroid-centered rotating frame, where $x$ and $z$ axes lie along the longest and shortest axis of the asteroid, respectively, and $x-y$ plane coincides with the asteroid rotation plane. The three semi-axes are $a, b$ and $c$ with $a \geqslant b \geqslant c$. The rotation period and angular velocity of the asteroid are $T$ and $\omega$. For simplicity, the motion of the asteroid around the Sun is assumed to be circular and coplanar with its own rotation and Sun-asteroid distance $r_{\mathrm{s}}$ is fixed to be 1 AU. Thus the dynamical problem is reduced to the planar case.

With the nondimentional length and time units taken as $a$ and $\tau=\omega t$, the geometry of the ellipsoid is $1: b / a: c / a=1: \beta: \gamma$ and angular rotation velocity of the Sun is $\omega_{\mathrm{s}}=1-n_{\mathrm{s}} / \omega$ in which $n_{\mathrm{s}}$ is the true rotation rate of the asteroid about the Sun. The equations of motion (EOM) are

$$
\left\{\begin{array}{l}
\ddot{x}-2 \dot{y}=\frac{\partial U}{\partial x}+\epsilon \cos (\varphi+\pi) \\
\ddot{y}+2 \dot{x}=\frac{\partial U}{\partial y}+\epsilon \sin (\varphi+\pi) .
\end{array}\right.
$$

The normalized magnitude of SRP is $\epsilon=\nu P \sigma r_{s}^{2} /\left(\omega^{2} a r_{s}^{2}\right)$ in which $\nu$ is the eclipse factor and the cylindrical eclipse model is adopted, $P$ is the solar pressure exerted on a satellite at $1 \mathrm{AU}$ and takes the value $4.56 \times 10^{-6} \mathrm{~N} \mathrm{~m}^{-2}$ and $\sigma$ is the area-to-mass ratio. The solar position angle is $\varphi=-\omega_{\mathrm{s}} \tau+\varphi_{0}$ with $\varphi_{0}$ serving as the initial value.

The effective potential is the same as defined by Scheeres (1994),

$$
U=\frac{1}{2}\left(x^{2}+y^{2}\right)-\delta V
$$

where $\delta=\mu /\left(\omega^{2} a^{3}\right)$ in which $\mu$ is the gravitational parameter of the ellipsoid and $V$ is the potential of the ellipsoid obtained from the integration

$$
V=\frac{3}{4} \int_{u}^{\infty} \phi(x, y, z ; v) \frac{\mathrm{d} v}{\Delta(v)}
$$

in which

$$
\begin{gathered}
\Delta(v)=\sqrt{(1+v)\left(\beta^{2}+v\right)\left(\gamma^{2}+v\right)} \\
\phi(x, y, z ; v)=\frac{x^{2}}{1+v}+\frac{y^{2}}{\beta^{2}+v}+\frac{z^{2}}{\gamma^{2}+v}-1
\end{gathered}
$$

and

$$
\phi(x, y, z ; u)=0
$$

\section{Dynamical Substitutes and Stability}

With the SRP taken into account, Eq. 2.1 becomes time-dependent and equilibrium points no longer exist. These equilibrium points turn into dynamical substitutes, which are periodic orbits around them. Obviously, the period of the dynamical substitute $T_{d}$ should be equal to that of the additional term arising from the SRP, i.e., $2 \pi / \omega_{s}$. We mainly focus on the dynamical substitutes for the center points in our following study. 


\subsection{Dynamical Substitute}

When SRP is small, the dynamical substitute orbits close enough to the equilibrium point and we can solve for the approximate solution of the dynamical substitute from the variational equations

$$
\left\{\begin{array}{l}
\ddot{\xi}-2 \dot{\eta}=p \xi-\epsilon \cos \varphi \\
\ddot{\eta}+2 \dot{\xi}=q \eta-\epsilon \sin \varphi
\end{array}\right.
$$

where $p=\partial^{2} U / \partial x^{2}$ and $q=\partial^{2} U / \partial y^{2}$ with respect to the center point $\left(x_{E}, y_{E}\right)$. The solution is

$$
\left\{\begin{array}{l}
\xi=A_{1} \cos \varphi+B_{1} \sin \varphi \\
\eta=A_{2} \cos \varphi+B_{2} \sin \varphi
\end{array}\right.
$$

and the coefficients are generally

$$
\left\{\begin{array}{l}
A_{1}=\frac{q-1}{p q+p+q-3} \epsilon \\
B_{2}=\frac{p-1}{p q+p+q-3} \epsilon \\
A_{2}=B_{1}=0 .
\end{array}\right.
$$

when the condition $p q+p+q-3 \neq 0$ holds. The constraint functions for the dynamical substitute are generally

$$
\left\{\begin{array}{l}
x\left(T_{d}+t_{0}\right)=x\left(t_{0}\right) \\
y\left(T_{d}+t_{0}\right)=y\left(t_{0}\right) \\
\dot{x}\left(T_{d}+t_{0}\right)=\dot{x}\left(t_{0}\right) \\
\dot{y}\left(T_{d}+t_{0}\right)=\dot{y}\left(t_{0}\right) .
\end{array}\right.
$$

We adopt differential correction method to numerically solve the constraint functions Eq. 3.4 for the initial state of the dynamical substitute with

$$
\left\{\begin{array}{l}
x\left(t_{0}\right)=x_{E}+\xi\left(t_{0}\right) \\
y\left(t_{0}\right)=y_{E}+\eta\left(t_{0}\right) \\
\dot{x}\left(t_{0}\right)=\dot{\xi}\left(t_{0}\right) \\
\dot{y}\left(t_{0}\right)=\dot{\eta}\left(t_{0}\right)
\end{array}\right.
$$

serving as the initial guess. We generally require the discontinuity between the final and the initial states does not exceed $10^{-11}$ in nondimensional unit. The initial guess is only valid for small SRP perturbation. For the larger SRP, we obtain the dynamical substitutes through continuation method. Figure 1 shows a family of dynamical substitutes under the effect of increasing SRP. The red ellipse in the figure represents the $x-y$ projection of the ellipsoid.

\subsection{Stability of Dynamical Substitutes}

We analyse the stability of the dynamical substitutes by evaluating the eigenvalues of the associated monodromy matrix. For the periodic dynamical substitute, the monodromy matrix is defined as the state transition matrix (STM) over one period. Generally, the eigenvalues of the monodromy matrix come in pairs which are conjugate as well as 


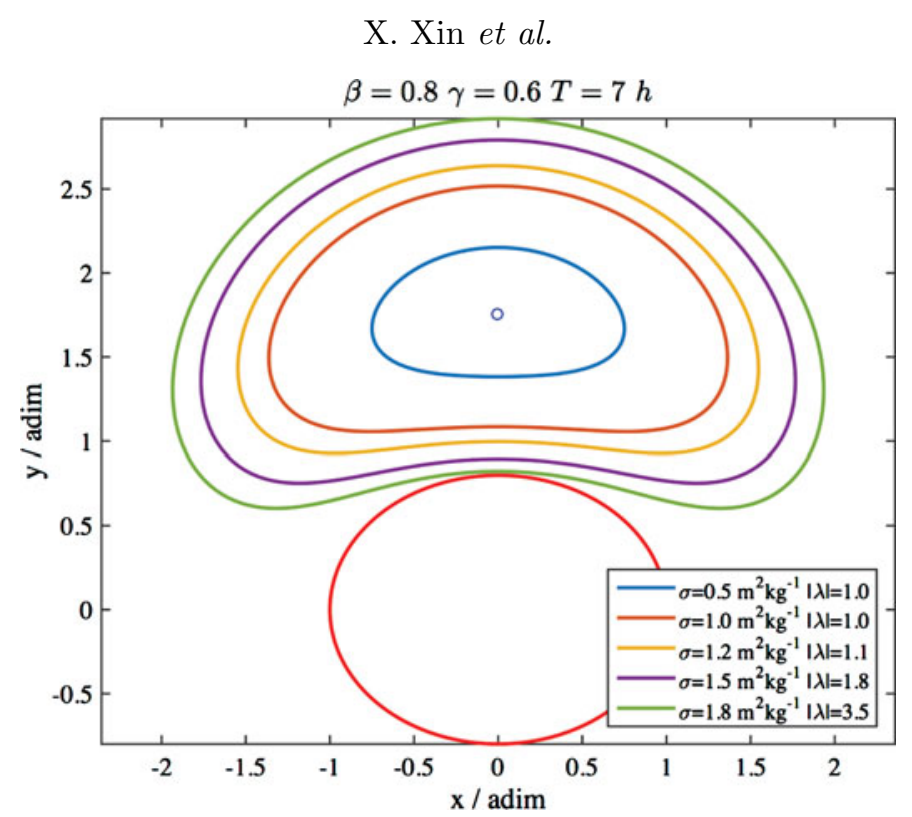

Figure 1. A family of dynamical substitutes. The red ellipse is the projection of the ellipsoid

reciprocal to each other. For stable dynamical substitute, eigenvalues take the form

$$
e^{ \pm \mathrm{i} k_{1}}, e^{ \pm \mathrm{i} k_{2}}
$$

in which $k_{1}$ and $k_{2}$ are real and all the modules of the eigenvalues are equal to one. For the unstable dynamical substitute, eigenvalues take the form

$$
\lambda, \frac{1}{\lambda}, e^{ \pm \mathrm{i} k}
$$

in which $\lambda$ and $k$ are real and $|\lambda|>1$ or

$$
\lambda, \bar{\lambda}, \frac{1}{\lambda}, \frac{1}{\bar{\lambda}}
$$

in which $\lambda$ is complex and $|\lambda|>1$.

For our dynamical model (Eq. 2.1), there are four free parameters, i.e., $\beta, \gamma, \sigma, T$. With each pair of $(\beta, \gamma)$, the selection of a pair of $(\sigma, T)$ will yield one dynamical substitute. We search the parameter space of $(\sigma, T)$ to obtain the stability map of the dynamical substitutes for certain ellipsoid shape $(\beta, \gamma)$, which we select four pairs, i.e., $(0.9,0.6)$, $(0.8,0.6),(0.7,0.6)$ and $(0.6,0.6)$. Fig. 2 shows the four stability maps. The blue and red regions corresponds to the parameter space where the dynamical substitute is stable and unstable, respectively. The white space left is the parameter space where either the dynamical substitute intersects with the ellipsoid or the corresponding center point lies inside the ellipsoid, the case when we assume the dynamical substitute no longer exists.

One unique feature in all four stability maps is the red gap in the stable blue regions. We specially designate the rotation period where the base of the gap lies at the "resonant rotation period" for which the values are listed in Table 1. At this rotation period, the smallest magnitude of the SRP is needed to achieve the transition for the dynamical substitutes from stable state to unstable state. This is due to the effect of the 2:1 resonance between the frequency of the external SRP $\omega_{s}$ and the intrinsic frequency of the center point. 

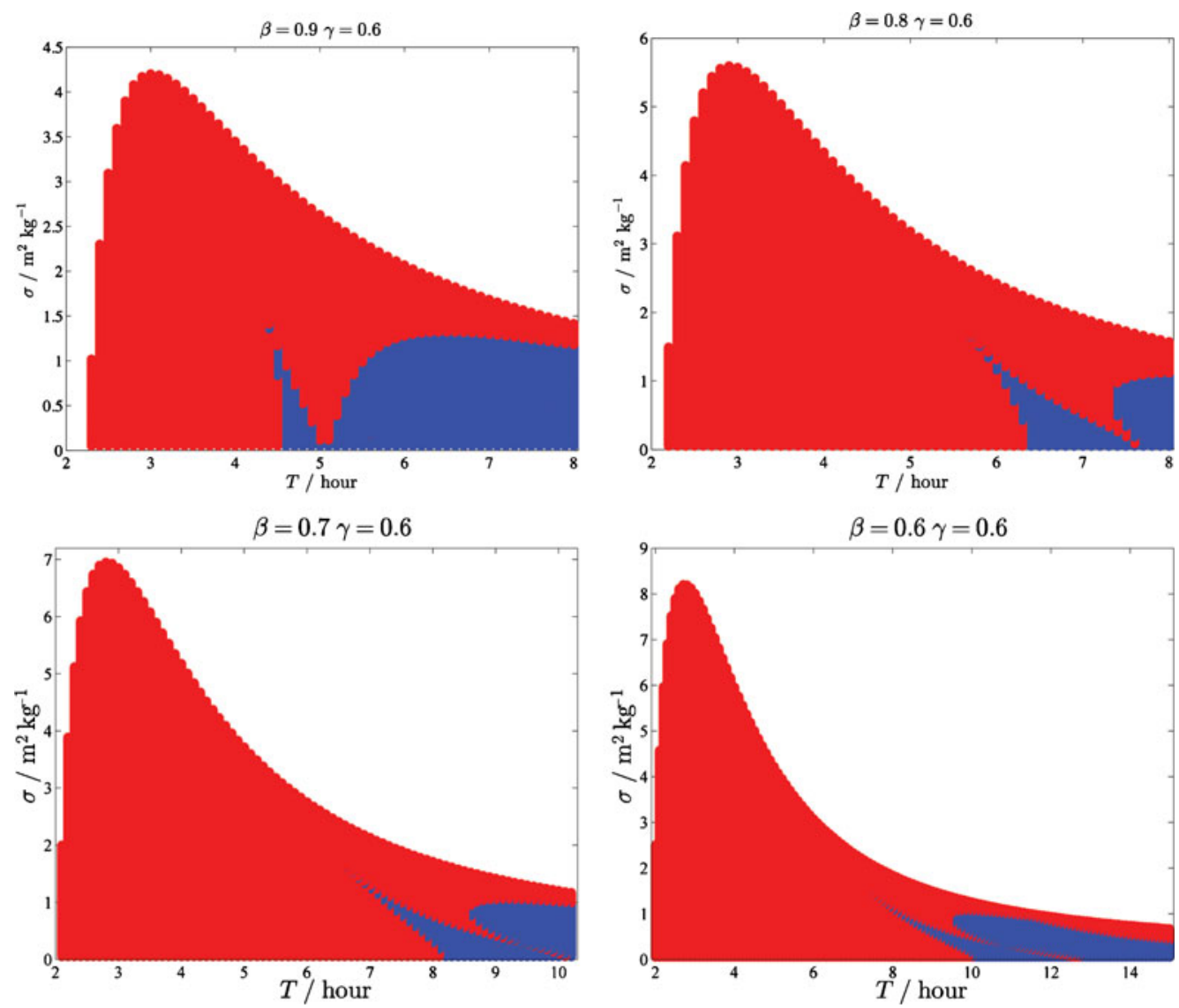

Figure 2. Stability maps of dynamical substitutes of different ellipsoid shapes. The blue and red regions corresponds stable and unstable dynamical substitute, respectively. The white regions left is the parameter space where either the dynamical substitute intersects with the ellipsoid or the corresponding center point lies inside the ellipsoid

Table 1. Rotation periods for different shapes of ellipsoid

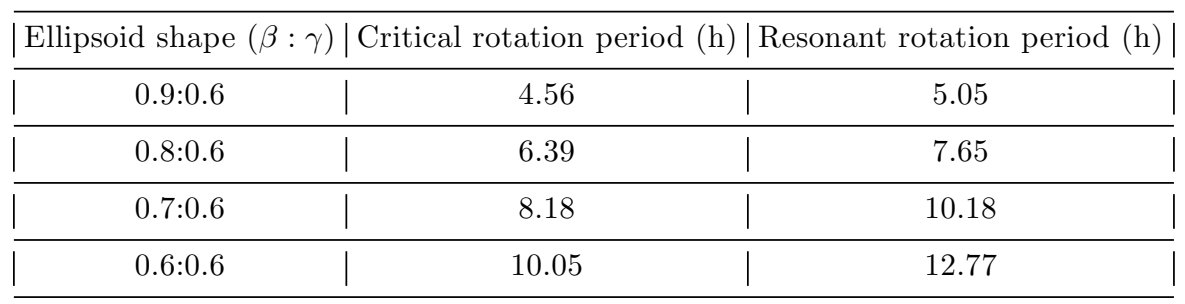

Another feature is that there exist these blue stable regions in all four figures where the rotation periods are smaller than the corresponding "critical rotation periods", below which the center points become unstable. This indicates that for ellipsoids with certain rotation periods, we can actually find the stable dynamical substitutes for the corresponding unstable center points. 


\section{Conclusion}

We model the asteroid as a uniformly rotating triaxial ellipsoid, and considering the effect of SRP perturbation, we compute families of dynamical substitutes, which are equivalent to the equilibrium points in the time-invariant dynamical system.

Different geometries and rotation periods of the asteroids as well as the magnitudes of the SRP perturbation are taken into account and how those factors influence the stability of the dynamical substitutes are investigated and shown in the stability maps. Two features clearly stand out: one is the steep drop at the resonant rotation periods when the smallest magnitude of SRP is needed for the stability transition of the dynamical substitutes; another is the existence of stable regions for dynamical substitutes of asteroids with rotation periods below the critical rotation period when the associated center points become unstable.

To better understand the dynamics of equilibrium points and dynamical substitutes of asteroid modelled as ellipsoid, future work should extend the numerical computation to 3D case and the effect of out-of-plane SRP perturbation needs to be accounted for in the dynamical model.

\section{References}

Morrow, E., Scheeres, D. J., \& Lubin, D. 2001, J. Spacecraft Rockets, 38, 279

Scheeres, D. J. 1994, Icarus, 110, 225

Scheeres, D. J. 2012, J. Guid. Control Dynam., 35, 987 